\section{Three-Dimensional Materials Science: An Intersection of Three-Dimensional Reconstructions and Simulations}

\section{Katsuyo Thornton and Henning Friis Poulsen, Guest Editors}

\begin{abstract}
The recent development of experimental techniques that rapidly reconstruct the three-dimensional microstructures of solids has given rise to new possibilities for developing a deeper understanding of the evolution of microstructures and the effects of microstructures on materials properties. Combined with three-dimensional (3D) simulations and analyses that are capable of handling the complexity of these microstructures, 3D reconstruction, or tomography, has become a powerful tool that provides clear insights into materials processing and properties. This introductory article provides an overview of this emerging field of materials science, as well as brief descriptions of selected methods and their applicability.
\end{abstract}

\section{Introduction}

Enabled by advances in experimental techniques and computational resources, a paradigm shift has transpired in materials science during the past decade. Traditionally, the methods of choice for structural characterization have been optical and electron microscopies, in which information is derived from two-dimensional (2D) sections. Today, the development of automated serial sectioning techniques and the exploitation of synchrotron radiation have made three-dimensional (3D) and even four-dimensional (4D, i.e., time- and space-resolved) studies possible. Simultaneously, the rapid increase in computational power, doubling approximately every two years, has enabled computational materials researchers to tackle much more complex problems, including large-scale 3D simulations of microstructure evolution, ties of complex microstructures cannot be assessed at all through stereology, including those related to morphology (e.g., the 3D shape of grains) and topology (e.g., the connectivity among microstructural features). Furthermore, observations on 2D sections might not be representative of the bulk because the observed phenomena can be affected in some manner by the presence of the free surface of the section.

Computationally, the change in dimensionality can yield very different results in simulated mechanical responses, microstructure evolution, and transport properties. As an example, because the functional form of the solution for the steady-state diffusion field depends on the dimensionality, the diffusion and resulting microstructural evolution under the assumption of two dimensions are quite different from those obtained for three dimensions. In addition, the connectivities of $2 \mathrm{D}$ and $3 \mathrm{D}$ microstructures can be quite different, which also influences the evolution. Figure 1 shows the micro-structural evolution resulting from coarsening following spinodal decomposition in systems with two phases, each with a volume fraction of $50 \%$. It shows that the 2D microstructure develops isolated domains of a phase that are fully embedded in the other phase (i.e., particles), whereas the 3D microstructure maintains bicontinuity (every part of each of the two phases is connected throughout the microstructure), even though the only difference between the two systems is their dimensions. Moreover, because transport properties depend significantly on the connectivity of the transporting medium, the difference in microstructural geometry and connectivity between the different dimensions creates a major gap in the predictions.

The combination of the new 3D and $4 \mathrm{D}$ characterization techniques and largescale simulations based on 3D data sets can overcome the limitations just mentioned and allow for a direct visualization of the microstructural evolution and materials responses that depend on the microstructure. Such information opens the door for a new generation of physicsbased models and enables much more realistic assessments of many technologically important materials with complex, heterogeneous microstructures. Computationally, because of the relatively large spatial and time scales involved in these structures and processes, continuum-level simulations at the scale of microstructures, rather than at the discrete individual-atom level, have found a dominant role so far.

In this issue of MRS Bulletin, we review selected pioneering work that illustrates 
the synergy between 3D simulations and experiments, focusing mainly on studies of microstructures in millimeterto centimeter-sized samples of optically opaque materials with a spatial resolution on the micrometer scale. Other techniques are briefly mentioned in this introductory article.

\section{Three-Dimensional Computational Materials Science}

Most materials comprise complex heterogeneous microstructures, and in many cases, they cannot be accurately studied by an analytical approach. Simulations must then be employed. Because of limited computational resources, simulations were first performed in lower dimensions (i.e., one and two dimensions). These simulations often provided insights and trends, but because of the lower dimensionality, they could not provide quantitative predictions. Two-dimensional simulations remain important tools in situations where the assumption of only two dimensions is valid (e.g., simulations of thin films) or where 3D simulations remain a challenge.

Unlike the case for experimental techniques, where changing the number of dimensions may require new methodologies, changing dimensionality in many types of simulations is relatively straightforward algorithmically. For typical partial differential equation simulations based on finite-difference methods, a change in dimension might merely involve adding an extra dimension in the arrays over which additional calculations must be made. For finite-element-based models, the extension to higher dimensions requires new algorithms to handle more complex elements and their connectivities, but the necessary tools have already been developed. Therefore, the challenge is often keeping the computational time and memory requirements within technological reach. In recent years, 3D simulations have become increasingly practical as a result of the ample availability of parallel computing, which allows for both computational time and memory. This increase in computing power has resulted in a revolutionary change in the manner in which materials science problems are tackled. Examples of such simulations are highlighted in the articles in this issue.

One of the goals of computational materials science is to establish the link between processing or operating conditions and resulting microstructures; see Figure 2. Because many microstructures are demarcated by their internal interfaces (e.g. between liquid/solid and gas phases, between regions with different atomic
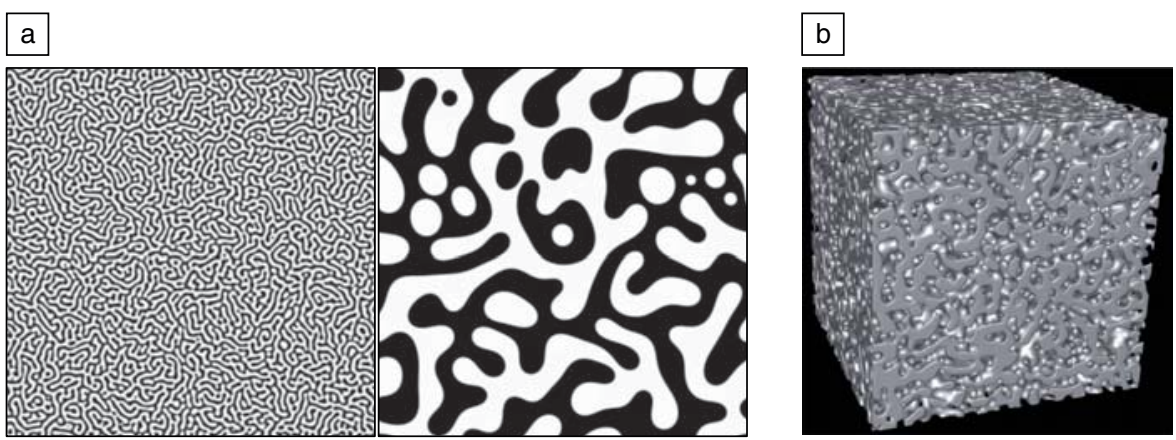

Figure 1. Illustration of differences between two-dimensional (2D) and three-dimensional (3D) simulations of microstructural evolution following spinodal decomposition with half of the material belonging to one phase (white) and half the other (black). (a) An early 2D microstructure is complex and has extensive connectivity over the characteristic length scale of the microstructure (top). However, as coarsening proceeds, the connectivity over the characteristic length scale decreases, and clearly isolated regions (particles enclosed in the other phase) are formed (bottom). (b) In three dimensions, the microstructural evolution following spinodal decomposition proceeds without changes in connectivity over the characteristic length scale of the microstructure. This figure is adapted from Reference 20.

\section{Processing

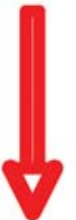 \\ Phase-field models \\ Level-set methods \\ Interface-tracking methods}

\section{Microstructure}

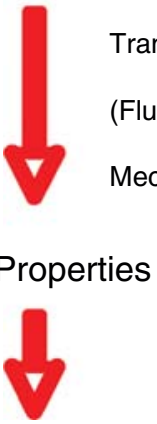

\section{Performance}

Figure 2. Schematic showing how simulations fit into the materials science and engineering framework of processing-structure-property-performance. (a) Threedimensional phase-field simulation result for ferrite nucleation and growth from the austenite phase in carbon steel from the article by Jensen et al. in this issue. (b) Finiteelement-modeling simulation of mechanical responses of a polycrystalline Ti alloy based on experimentally measured 3D microstructures from the article by Spanos et al. in this issue; von Mises stress is shown.

structure, or between grains with different orientations), techniques for simulating microstructural evolution typically require a method for either interface tracking (by moving mesh points on the interface) or interface capturing (by evolving a spatially varying function that defines the location of the interface). Whereas interface tracking is relatively straightforward in lower dimensions, it poses significant difficulties in three dimensions, and thus, interfacecapturing methods have gained popular- 
ity in recent decades as 3D simulations have become more tractable.

Interface-capturing methods include level-set methods and phase-field methods. In the level-set method, ${ }^{2}$ the spatially varying function could be the signed distance from the closest interface (positive on one side, negative on the other), and thus two bulk regions have either positive or negative values of this function. The interface is then given by the location where the function interpolates to zero (i.e., the zero level set). The evolution of this function can be described by considering the governing physics of interfaces such as the motion induced by interfacial tension. An alternative approach, phasefield modeling, ${ }^{3}$ has a constant, predetermined value of the function in each bulk phase/grain orientation. Thus, the function varies only in the interface, which defines the interfacial region. As discussed in the article by Kammer et al., this method is formally based on thermodynamic treatment of systems undergoing spinodal decomposition. Recently however, it has found more general application as a computational method for simulating microstructural evolution in a wide range of materials systems. ${ }^{3}$ Because microstructural evolution is a complex phenomenon governed by thermodynamics, kinetics, and in some cases even mechanics, the development and incorporation of the correct models remains an active focus of research. The phase-field approach has been extensively utilized in the area of solidification and dendrite formation where it has become a tool that allows quantitative predictions. Other methods, including those based on interface tracking, have also found applications in materials science and can be more suitable than phase-field modeling in some problems.

Another aspect of computational materials science is the linking of microstructure to materials properties (see Figure 2). In this area, simulations using finiteelement-based tools have been employed to examine the mechanical and transport properties of 3D microstructures in materials. This type of modeling is well established, ${ }^{4}$ and many tools are commercially available (e.g., Fluent and COMSOL). What had been lacking in this area were the tools to incorporate real 3D data into the finite-element mesh. This is still challenging when the structure to be imported is large and complex, but it is becoming easier with new releases of software designed to handle such tasks (e.g., Mimics and 3-matic).

Whereas this issue focuses mainly on continuum-level simulations, simulation methods based on discrete, atomic-level descriptions of materials, such as molecular dynamics and kinetic Monte Carlo, are commonly applied to materials science problems. These methods involve the tracking of each atomic position and allow interfaces to be modeled by having regions with different compositions or crystallographic orientations. Although these methods cannot simulate systems on a large scale, they provide essential information regarding the atomic-scale bonding, ordering, and interactions in materials. They can also simulate a scaleddown system, which can be used to infer materials behavior at a larger scale. Such an example is given for molecular dynamics in conjunction with 3D experimental work in the article by Jensen et al. in this issue. This article also includes a discussion of geometry-based modeling, which determines the grain structures based on geometrical restrictions rather than the physical interactions that drive grain boundary evolution.

Materials phenomena are fundamentally multiscale; small-scale physics (electronic structures defining atomic interactions, which determine atomic order, distributions, and transport) affects the evolution at the microstructural level during processing and material responses during usage. Advances in modeling efforts have been hindered by a lack of complete experimental information to evaluate, improve, and validate the input physics and parameters in the models. The recent developments in $3 \mathrm{D}$ and $4 \mathrm{D}$ experimental techniques now provide new opportunities to build quantitative, predictive models for a wide range of materials and processes.

\section{Three-Dimensional Experimental Materials Science}

Three-dimensional reconstruction of structures from a series of $2 \mathrm{D}$ sections is conceptually the simplest of all of the 3D experimental techniques. As shown in Figure $3 \mathrm{a}$, in this approach, the images of 2D slices that are equally (or nearly equally) spaced are obtained consecutively. A 3D map is then obtained by interpolation. (For more detail, see the articles by Spanos et al. and Kammer et al. in this issue.) For opaque materials, this requires removal of a layer of material from the sample to obtain each of the 2D images. Thus, this technique is fundamentally destructive. The feature sizes that can be analyzed depend on the resolution along the sectioning direction, which is limited by the fact that it becomes increasingly difficult to remove a precise thickness of material as the sectioning thickness decreases. Using a traditional milling method (such as diamond blades ${ }^{6}$ or diamond lapping films ${ }^{7}$ ), this limit is approximately $0.1 \mu \mathrm{m}$ at best and is often greater than $1 \mu \mathrm{m}$. Another limitation in this approach is that, even with automation, the number of $2 \mathrm{D}$ sections that can be obtained is currently $\sim 20$ per hour. If the integrated imaging instrument (i.e., microscope) is an optical one, the two other directions of the investigated volume are typically defined by the field of view of the microscope. Hence, the reconstructed volume tends to be thinner along the sectioning direction.

An alternative serial-sectioning-based method uses dual-beam microscopes equipped with a focused ion beam (FIB) gun and secondary-electron detectors, which recently have become commercially available. For a recent review, see Reference 8. In this method, 3D maps are generated automatically by alternate ionbeam milling and imaging sessions. If an electron backscatter diffraction (EBSD) system is combined with such equipment, one can obtain a 3D map of the crystallographic orientation. The size of the investigated volume is limited by the FIBmilling rate, but volumes as large as 50 $\mu \mathrm{m}^{3}$ have been reported with 50 -nm resolution. For an overview of this and other techniques for 3D characterization of nanostructures (such as the 3D atomprobe microscopy, nano-x-ray tomography, and phase-retrieval methods), see Reference 9.

The current probe of choice for nondestructive 3D mapping of opaque materials is $x$-rays in the $20-200 \mathrm{keV}$ range. With such a beam, it is possible to penetrate up to $5 \mathrm{~mm}$ of steel, $4 \mathrm{~cm}$ of aluminum, or $10 \mathrm{~cm}$ of polymer-based materials. When generated by a synchrotron source, the beam is also highly collimated and ordersof-magnitude more intense than that obtained with conventional x-ray sources. The gain in intensity translates into a corresponding improvement in spatial or temporal resolution. Within the past decade, the number of beamlines dedicated to 3D mapping applications has grown rapidly to $\sim 30$ worldwide. This trend is continuing, with new regional synchrotrons in the planning stage or in construction.

Absorption contrast tomography is the oldest imaging technique and the only one that is readily applicable to conventional x-ray sources as well. ${ }^{10,11}$ In situ experiments are possible because the $\mathrm{x}$-rays can penetrate furnaces and other equipment surrounding the sample. As illustrated in Figure 3b, a 3D density map is reconstructed by an inverse algorithm from a set of typically $1,0002 \mathrm{D}$ projection 
a Sectioning

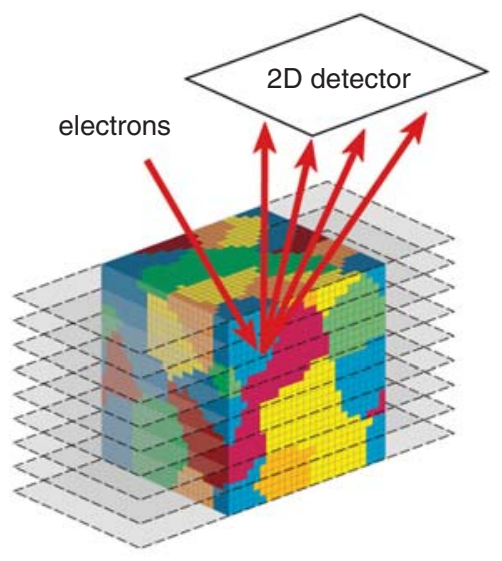

b Tomography x-rays

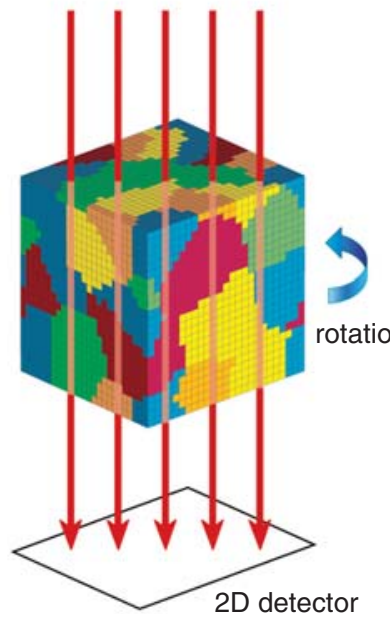

C 3DXRD microscopy x-rays

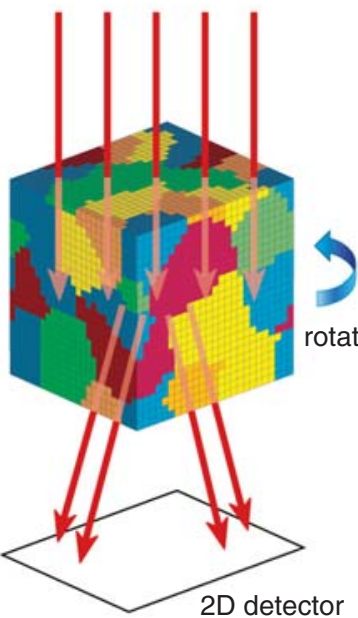

d X-ray scanning x-rays

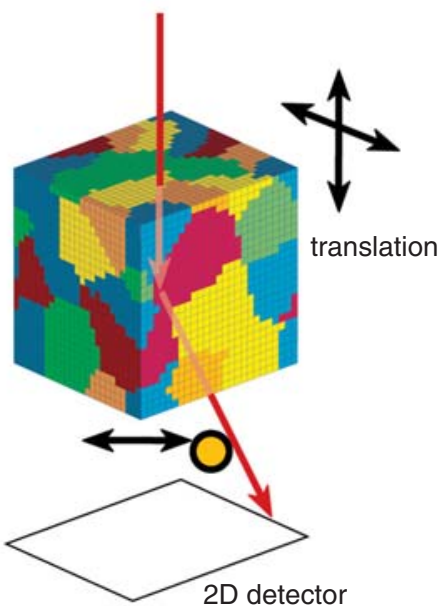

Figure 3. Principles of 3D experimental methods. (a) Sectioning: Direct construction of a 3D map by interpolation along the normal axis of a set of 2D maps. The sections are made by mechanical polishing 6,7 or by a focused ion beam. ${ }^{8}$ (b) X-ray tomography: The $\mathrm{x}$-ray beam illuminates the full sample, and the attenuation of the beam is projected onto a 2D detector. From a set of such 2D projection images, the 3D density field is reconstructed.10 (c) Three-dimensional x-ray diffraction microscopy: The setup is similar to that in (b), but the images are obtained from multiple sets of diffraction spots rather than attenuated intensities. Based on algorithms from mathematical tomography, 3D grain-orientation maps are generated. ${ }^{14}$ (d) X-ray microbeam scanning: The diffraction signal is probed voxel by voxel to generate 3D maps of the orientation and stress fields. ${ }^{15}$ The orange dot represents an absorbing wire.

images acquired while rotating the sample about one axis. Three-dimensional reconstructions comprising up to $1,000 \times$ $1,000 \times 1,000$ voxels are now routinely made. (A voxel is the smallest elementary volume element composing 3D images.) The data acquisition time for a complete 3D tomogram has recently been pushed into the range of seconds. ${ }^{12}$ The main limitation is the density contrast. Using coherent x-ray beams (having the same phase), which are now available at synchrotrons, the detectable density contrast can be improved to $\sim 0.05 \mathrm{~g} / \mathrm{cm}^{3}$ by socalled phase-contrast methods. ${ }^{13}$ In this approach, the image contrast is improved because the beam's phase can change measurably as the beam propagates a sample even if the contrast due to attenuation is too small to be detected.

Tomography cannot characterize the grain structure in a single-phase sample, as the density is constant. This limitation is overcome by three-dimensional $x$-ray diffraction (3DXRD) microscopy, which applies to polycrystalline specimens ${ }^{14}$ (see Figure 3c). The setup and reconstruction approach is similar to those for tomography, but one probes the diffracted beam rather than the attenuation of the incoming beam. Through analysis of the diffraction patterns, grain orientations are obtained as a function of position; the result is a 3D orientation map. 3DXRD also provides infor- mation on the local stress state and the local phase (atomic composition and/or structure). The spatial resolution is $5 \mu \mathrm{m}$ for a complete 3D map. However, the microscope can be run in other modes, thereby enabling studies of the change in volume, orientation, and stress of hundreds of individual embedded grains as small as $20 \mathrm{~nm}$ with a time resolution of seconds. (See the article by Jensen et al. in this issue and the references therein.)

As an alternative to the imaging techniques mentioned thus far, where the complete sample is illuminated, one can probe the crystalline structure voxel by voxel by focusing the $x$-ray beam to essentially one ray (Figure 3d). To determine the depth of origin of a certain diffraction signal, the diffracted beam is confined by a slit, a collimator, or (as shown in Figure 3d) an absorbing wire. By scanning the sample in two directions perpendicular to the beam and by scanning the confinement with respect to the beam, one can generate $3 \mathrm{D}$ maps of the orientations or stresses. Scanning techniques are, by nature, slower than imaging approaches, and the need for absorption elements in proximity to the sample could limit options for in situ work. On the other hand, the spatial resolution can be better, as it is determined by the specifications of $\mathrm{x}$-ray optics rather than $\mathrm{x}$ ray detectors. As an example, a polychromatic setup at the Advanced Photon
Source enables 3D orientation and strain maps to be acquired with a resolution of better than $500 \mathrm{~nm} .{ }^{15}$ Scanning techniques are also used widely on the engineering scale to acquire maps of the 3D residual stress fields in structural materials such as rail tracks. ${ }^{16}$ (For a comparison of all available $\mathrm{x}$-ray-based techniques, see also the March 2004 issue of MRS Bulletin. ${ }^{17}$ )

Neutron equivalents of the tomography $^{18}$ and strain scanning techniques ${ }^{16}$ are also used widely. The spatial resolution is two to three orders of magnitude worse, but typically one can probe larger samples.

The experimental methods described so far are complementary in terms of space and time resolutions, contrast mechanism, size of sample, and options for in situ work. Hence, it is expected that many investigations will combine multiple techniques in order to build more complete knowledge of materials phenomena. Furthermore, all techniques tend to be limited by detector technology or computing power, both of which are rapidly evolving. Hence, it is reasonable to predict that data acquisition rates can improve by two orders of magnitude within the next decade.

\section{Time-Dependent Three- \\ Dimensional Materials Science}

An advantage of computational investigations of materials processes is that timedependent information is often generated 
naturally. The prediction of microstructural evolution is typically made by calculating the change in microstructure over a very small duration of time (the so-called time step), updating the microstructure, and then repeating this process. In such cases, one can observe how a certain process proceeds and thus determine what mechanisms possibly play a role in experimentally observed phenomena. For example, by making a movie of the evolution of a microstructure consisting of particles, it is possible to determine whether the average particle size grows solely due to diffusion or whether other mechanisms, such as coalescence, are involved.

Experimentally, adding the time dimension is frequently challenging. First, the sample must be observed under the conditions that induce the desired changes. Thus, a microscope or other types of detector must be integrated with equipment that sets the experimental conditions (e.g., furnaces or mechanical or tensile machines) to allow in situ data collection. Second, the sample must not be destroyed during the data collection. Third, data collection must be fast enough to provide the desired time resolution. The latter two requirements pose a major challenge in $3 \mathrm{D}$ experimental investigations. As mentioned previously, the recent advances in 3D experimental techniques based on $x-$ ray tomography and diffraction have enabled nondestructive measurements of microstructures with sufficient time resolution (see Figures $3 b$ and $3 c$ ). The resulting knowledge not only offers clear insights into dynamic processes occurring within materials but also provides validation data for modeling and simulations, as discussed in the following section.

\section{Integration of Experiments and Simulations}

The first scientific results emerging from 3D and 4D experimental work have typically arisen from simple observations of maps or movies, demonstrating the violation of basic assumptions in conventional models. Studies with direct integration of 3D experiments and 3D simulations are still scarce (see the accompanying articles in this issue for examples), but they provide examples for a range of new approaches for materials investigations. There are several modes of integration of 3D experimental and computational materials science, which can be categorized as follows:

- Direct examination of assumptions. Simple observations of microstructures or their evolution have supported or ruled out common assumptions made in model- ing. (See, for example, the article by Jensen et al. in this issue, which provides an example of a case where the assumption that all grains have the same growth rate during recrystallization is violated.)

- Experimental acquisition of statistical data for input to models or simulations. Such statistical data can be in various dimensions. For example, the nucleation rate per unit volume as a function of time is one-dimensional. At the other extreme, the nucleation rate as a function of time and region (e.g., bulk or interfaces) is $4 \mathrm{D}$. The data can also be a function of morphological characteristics or features, such as the particle size distribution in particulate microstructures or corners in polycrystalline microstructures.

- Experimental acquisition of statistical data for validation of models and simulations or, in some cases, for validation of other experimental techniques (e.g., those based on 2D sections).

- Use of experimentally determined 3D microstructures as input to simulations (Figure 2b). This allows more accurate predictions of evolution, responses, or properties that are sensitive to the 3D microstructures. For example, artificially created microstructures are often based on simple inputs such as particle size distributions and volume fractions; therefore, they can have vastly different connectivities or correlations that can directly alter the resulting properties or evolution.

- Experimental acquisition of 4D data for direct 4D comparisons with simulation results. When a 3D structure is available, simulations can employ it directly to predict how the material might evolve or respond under given experimental conditions. The results can then be compared, voxel by voxel, to determine whether the prediction matches with the experimentally observed changes, providing a very stringent test of models. Such comparisons are now possible with $\mathrm{x}$-ray-based in situ techniques.

- Validation of experimental testing outside normal conditions. In some cases, experimental conditions are altered to extrapolate materials behavior. If the process under consideration is too slow, it could be accelerated by, for example, increasing the temperature. (This is termed accelerated testing and is often needed for materials with very long lifetimes.) In other cases, the extreme conditions of interest are not replicable in a given laboratory setting, requiring inferences from experiments under very different conditions. The combination of 3D experiments and 3D simulations provides a feedback loop to refine and validate both aspects.
All 3D experimental and modeling tools currently are limited to volumes of approximately $1,000 \times 1,000 \times 1,000$ voxels or less. For many problems in materials science, this is not sufficient to provide a full description of all mechanisms underlying the phenomena. On the simulation side, a major effort has been undertaken to facilitate multiscale modeling (see, for example, the November 2007 issue of MRS Bulletin ${ }^{19}$ ) where the direct linkage of models ranging from quantumphysics-based electronic calculations to continuum simulations could be made. Similarly, multifaceted efforts will likely emerge on the experimental side. For example, by combining a nondestructive experimental technique such as 3D x-ray imaging on a coarse scale, FIB-based 3D reconstruction on a finer scale, and $3 \mathrm{D}$ atom-probe microscopy at an even finer scale, one has an opportunity to capture materials phenomena over six orders of magnitude in length scale. This will bring materials researchers closer to the ultimate dream of a direct validation of multiscale models, both component by component and ultimately as an integrated simulation tool. In conjunction with the advances on the modeling side, such comprehensive experimental information is seen as very promising for establishing a new generation of models in materials science based on first principles.

\section{In This Issue}

The remainder of this issue comprises four articles that highlight state-of-the-art techniques and recent advances in which 3D and 4D experimental investigations of microstructures are directly linked to theory or simulations, many of which are computationally intensive. The focus on the microstructural length scale was chosen in order to provide an in-depth coverage of this subject. Therefore, detailed discussions of experimental techniques such as 3D atom-probe microscopy and atomic-/molecular-level simulation techniques are beyond the scope of the issue. Two main types of experimental techniques, namely those based on $x$-rays and those based on serial sectioning, are evenly divided into two sets of articles, which also provide focused discussions of accompanying simulation techniques. The article by Spanos et al. focuses on relating the microstructure and mechanical properties in polycrystalline systems through serial sectioning using the FIB, along with the EBSD technique that provides information on the grain orientation. Threedimensional finite-element modeling using the 3D data describes the mechanical response and, ultimately, the 
microstructure-property relationship (see Figure 2). The second article by Kammer et al. focuses on a serial sectioning technique using mechanical milling on the experimental side. It provides information on such techniques and describes new types of characterization and analysis that can be performed on 3D structures. The work described aims to understand microstructural evolution by combining the 3D experimental results with phasefield simulations, thereby providing a link between processing and/or operating conditions and microstructures (see Figure 2). The remaining two articles are based on synchrotron x-ray studies. Both articles highlight the advantage of in situ measurements that can be obtained by these nondestructive techniques. For example, the article by Buffière et al. discusses x-ray tomography and use of the resulting data to model a wide range of materials problems including mechanical responses of cellular materials (e.g., metallic foams), solidification, and crack growth The article also includes a discussion of a technique that combines 3DXRD with tomography. In addition, the article by Jensen et al. provides examples in which 3DXRD is used in polycrystalline materials to allow model validation, to generate model input, and to provide physical insights in concert with simulations.

These articles together illustrate the fact that the tools for obtaining 3D data, as well as for simulating materials in three dimensions, are now at hand, at least at the length scales relevant to microstruc- tures. Efforts will continue to push the experimental resolution both in time and in space, as well as to improve the prediction accuracies of simulations, which can now be tested against 3D/4D data. On the other hand, the ability to take full advantage of the wealth of data resulting from both experimental and simulation work is still a distant goal. Thus, creating new knowledge and techniques designed to extract important information from complex data in three and four dimensions will be essential for further development of the field of three-dimensional materials science, bringing yet another interdisciplinary aspect to this endeavor.

\section{Acknowledgments}

K. Thornton gratefully acknowledges financial support from the National Science Foundation under Grants DMR0502737, DMR-0542619, DMS-0511232, and CMMI-0700301; the Department of Energy Office of Basic Energy Sciences under Grant DE-FG02-05ER46191; and the Air Force Office of Scientific Research under Grant FA9550-05-1-0416. H.F. Poulsen acknowledges the Danish National Research Foundation.

\section{References}

1. J.C. Russ, R.T. DeHoff, Practical Stereology (Kluwer Academic/Plenum Publishers, New York, 2000).

2. J.A. Sethian, Level Set Methods and Fast Marching Methods (Cambridge University Press, Cambridge, UK, 1999).

3. L.Q. Chen, Annu. Rev. Mater. Res. 32, 113 (2002).
4. T.J.R. Hughes, The Finite Element Method: Linear Static and Dynamic Finite Element Analysis (Dover, Mineola, NY, 2000).

5. D. Frankel, B. Smit, Understanding Molecular Simulation: From Algorithms to Applications (Academic Press, San Diego, CA, 2002).

6. J. Alkemper, P.W. Voorhees, J. Microsc. 201, 388 (2001).

7. J.E. Spowart, H.M. Mullens, B.T. Puchala, JOM 55, 35 (2003)

8. M.D. Uchic, L. Holzer, B.J. Inkson, E.L. Principe, P. Munroe, MRS Bull. 32, 408 (2007).

9. Mater. Today 10 (12) (2007)

10. J. Banhart, ed., Advanced Tomographic Methods in Materials Research and Engineering (Oxford University Press, Oxford, UK, 2008).

11. G.T. Herman, Image Reconstructions from Projections (Academic Press, New York 1980).

12. O. Ludwig, M. Dimichiel, L. Salvo, M. Suery, P. Falus, Metall. Mater. Trans. A 36A, 1515 (2005).

13. P. Cloetens W. Ludwig, J. Baruchel, D. Van Dyck, J. Van Landuyt, J.P. Guigay, M. Schlenker, Appl. Phys. Lett. 75, 2912 (1999).

14. H.F. Poulsen, Three-Dimensional X-Ray Diffraction Microscopy (Springer, Berlin, Germany, 2004).

15. B.C. Larson, W. Yang, G.E. Ice, J.D. Budai, J.Z. Tischler, Nature 415, 887 (2002).

16. W. Reimers, A.R. Pyzalla, A. Schreyer, H. Clemens, eds., Neutrons and Synchrotron Radiation in Engineering Science (Wiley-VCH, Weinheim, Germany, 2008).

17. MRS Bull. 29 (3) (2004)

18. P. Vontobel, E.H. Lehmann, R. Hassanein, G. Frei, Physica B 385, 475 (2006).

19. MRS Bull. 32 (11) (2007).

20. Y. Kwon, K. Thornton, P.W. Voorhees, Phys. Rev. E 75, 021120 (2007).

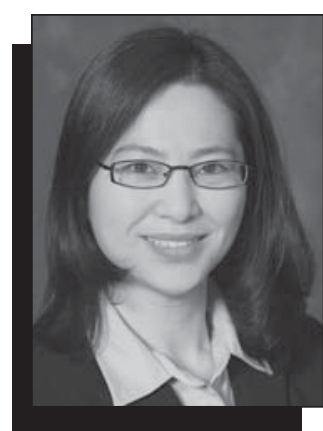

Katsuyo Thornton

Katsuyo Thornton can be reached at the Department of Materials Science and Engineering, University of Michigan, 2300 Hayward St., Ann Arbor, MI 48109, USA; tel. 734-615-1498; fax 734-

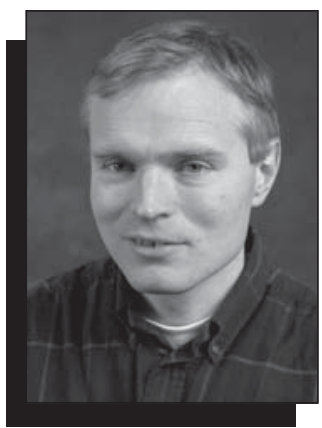

Henning Friis Poulsen

763-4788; e-mail kthorn@umich.edu.

Thornton, Guest

Editor for this issue of MRS Bulletin, is an assistant professor of materials science and engineering at the
University of Michigan, Ann Arbor. She received her $\mathrm{PhD}$ degree from the Department of

Astronomy and Astrophysics at the University of Chicago in 1997. Afterward, Thornton was a postdoctoral fellow at Northwestern University, and a visiting lecturer and scientist at the Massachusetts Institute of Technology, both in materials science and engineering. She then spent three years as a research assistant professor in materials science and engineering at Northwestern. Thornton's research focuses on computational studies of the evolution of microstructures and their effects on a wide range of materials including metals, semiconductors, ceramics, and biomaterials. She is the recipient of several prestigious awards including the TMS Early Career Faculty Fellow Award and the Carl Sagan Excellence in Teaching Award.

Henning Friis Poulsen can be reached at Materials Research Department, Risø DTU, Dk-4000 Roskilde, Denmark; tel. 45-46775739; fax 45-4677-5758 e-mail henning.friis. poulsen@risoe.dk.

Poulsen, Guest Editor for this issue of MRS Bulletin, is a research professor in the Materials Research Department at Risø DTU, the Technical University of Denmark. Poulsen earned his PhD degree in physics from the University of Copenhagen, Denmark, in 1991. Before joining Risø, he spent three years as a postdoctoral researcher at the HASYLAB synchrotron facility in Hamburg, Germany. At Risø, Poulsen heads the Centre for Fundamental Research: Metal 


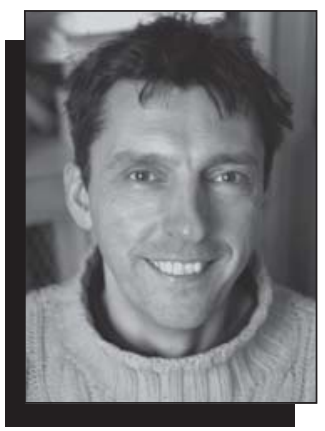

Jean-Yves Buffière

Structures in Four

Dimensions. His research interests are in the development of diffraction and imaging techniques based on high-energy $\mathrm{x}$-rays, and their application to materials science and engineering including plastic deformation and nucleation and growth phenomena. Poulsen has authored or co-authored more than 150 articles.

Jean-Yves Buffière can be reached by e-mail at jean-yves.buffiere@ insa-lyon.fr.

Buffière has been a professor at the Insitut National des Sciences Appliquées de Lyon (INSA Lyon) in France since 2003. Buffière first joined INSA Lyon as a lecturer in 1994. Since then, Buffière has been working on the characterization of damage in advanced materials under load. Through a collaboration with the European Synchrotron Radiation Facility, he has developed the use of high-resolution x-ray tomography for the nondestructive threedimensional (3D) characterization of materials. In addition, Buffière organized the first European workshop on that topic in 1999, and he has supervised or co-supervised several $\mathrm{PhD}$ projects and postdoctoral research

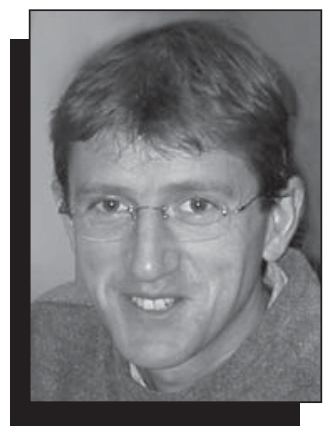

Peter Cloetens

programs in this field of research.

Peter Cloetens can be reached by e-mail at cloetens@esrf.fr.

Cloetens is a research scientist in the X-Ray Imaging Group at the European Synchrotron Radiation Facility. He obtained his $\mathrm{PhD}$ degree from the Department of Applied Sciences of the University of Brussels in 1999. Cloetens' main interest is quantitative 3D imaging, both in absorption and phase-contrast mode, applied to several topics in materials and life sciences. His recent work pursues the extension of hard x-ray imaging to the deep submicron domain by means of a nanoprobe. Cloetens implemented a high-resolution absorption and phase-contrast tomography end-station at the imaging beamline ID19, and a nano-imaging end-station for $\mathrm{x}$-ray fluorescence mapping and projection microscopy at beamline ID22.

Andrew B. Geltmacher can be reached by telephone at 202-767-6451; e-mail andrew. geltmacher@nrl.navy.mil.

Geltmacher joined the Naval Research

Laboratory in 1994, first as a National Research Council postdoctoral fellow, then as a contractor with FM Technologies Inc., and finally as a gov-

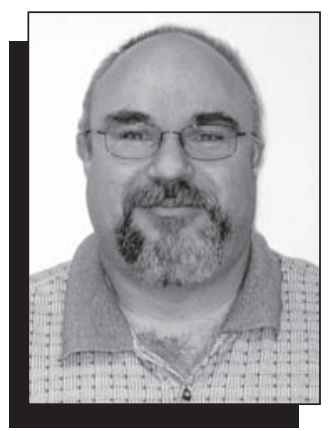

Andrew B. Geltmacher

ernment employee. He received his $\mathrm{MS}$ and $\mathrm{PhD}$ degrees in metals science and engineering from the Pennsylvania State University (PSU) in 1991 and 1994, respectively. Geltmacher received his BS degree with high distinction and honors in engineering science and mechanics from PSU in 1987. His research interests include engineering mechanics, material characterization, and mechanical behavior with special interest in microstructural effects on deformation and fracture.

Dorte Juul Jensen can be reached at the Materials Research Department, Frederiksborgvej 399, Box 49, 4000 Roskilde, Denmark; tel. 45-46775701; fax 45-4677-5758; e-mail dorte.juul.jensen @risoe.dk.

Jensen is the head of the Materials Research Department, Risø DTUNational Laboratory for Sustainable Energy, at the Technical University of Denmark. Jensen received her MSc degree in electrophysics from the Technical University of Denmark in 1980. She has been employed at Risø National Laboratory since 1983. From 2001 to 2006, Jensen was head of the Center for Fundamental Research: Metal Structures in Four Dimensions. Her main

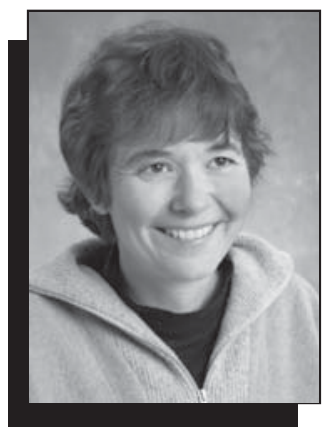

Dorte Juul Jensen

research interests include recrystallization, metal microstructures, plastic deformation, experimental characterization, electron microscopy, and neutron and x-ray diffraction. Jensen has received several Danish awards and has been a member of the Danish Academy of Technical Sciences since 1999. She also has been a principal editor of Scripta

Materialia since 2001 and has published more than 250 papers.

Dimitris Kammer can be reached at Northwestern University, 2220 Campus Dr., Evanston, IL 60208, USA; tel. 847-491-3425; e-mail dkammer@ mac.com.

Kammer is a postdoctoral researcher at Northwestern University. He received his diploma in metallurgical and mining engineering from the National Technical University of Athens in 2001. Also in 2001, Kammer joined the Department of Materials Science and Engineering at Northwestern, receiving his $\mathrm{PhD}$ degree in 2006. After fulfilling his military service requirement in his home country of Greece, Kammer returned to

Northwestern in 2007, serving in his current position. Kammer's research interests include dendritic coarsening,

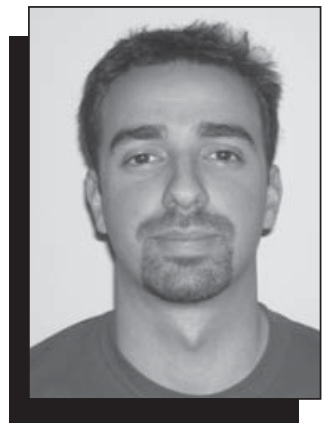

Dimitris Kammer

microstructural characterization, and morphological evolution.

Alexis C. Lewis can be reached by telephone at 202-767-3316; e-mail alexis.lewis@nrl.

navy.mil.

Lewis has been a staff scientist in the Multifunctional Materials Branch of the Naval Research Laboratory since 2005. She received her BS degree in 1997 from the Massachusetts Institute of Technology and her PhD degree in 2003 from the Johns Hopkins University, both in materials science and engineering. In 2003, she joined the Naval Research Laboratory as a U.S. National Research Council postdoctoral associate. Lewis' research interests center around advanced-materials characterization, including 3D analysis of the crystallography and mechanical response of materials. Lewis is a member of the Materials Research Society; The Minerals, Metals, \& Materials Society; and ASM International.

Wolfgang Ludwig can be reached by e-mail at ludwig@esrf.fr.

Ludwig is a research scientist for the National Center for Scientific Research, working in the MATEIS laboratory at INSA Lyon, France. He 


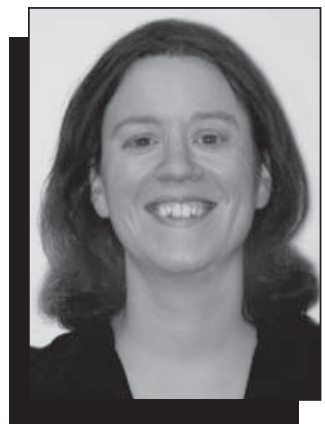

Alexis C. Lewis

received his $\mathrm{PhD}$ degree from the University of Munich in 2001.

Afterward, Ludwig spent one year as a postdoctoral researcher working in the field of x-ray transmission microscopy at the European

Synchrotron Radiation Facility (ESRF). He works at the ESRF on the development of diffraction contrast tomography, a new tomographic imaging technique combining the principles of $\mathrm{x}$-ray absorption and $\mathrm{x}$-ray diffraction imaging for nondestructive characterization of a materials 3D grain microstructure.

Eric Maire can be reached by e-mail at eric.maire@insa-lyon.fr.

Maire is a research associate working on microstructure and damage of bulk and cellular materials for the National Center for Scientific Research (CNRS) in the MATEIS Laboratory at INSA Lyon, France. Maire received his $\mathrm{PhD}$ degree in materials science in 1995 and spent one year as a postdoctoral researcher at McMaster University in Canada. He also is vice director of the RAMS federation of French laboratories and is strongly involved in the European group of research, HETMAT.

Maire has received sev-

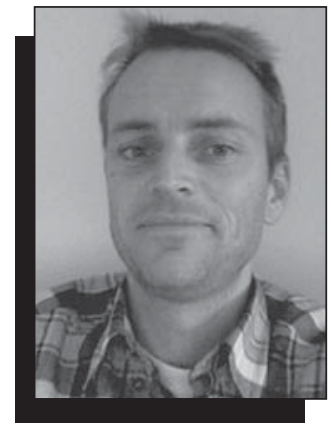

Wolfgang Ludwig

eral French and

European awards including the CNRS Bronze

Medal, the Jean Rist medal from the Société

Francaise de Metallurgie et de Matériaux, and the Alcan award of the French Academy of Science; and also has been honored as a

Federation of European Microbiological Societies lecturer. He is the coauthor of approximately 60 peer-reviewed articles in international journals and has co-organized five conferences.

\section{S. Erik Offerman can be} reached at the

Department of Materials Science and Engineering, Delft University of

Technology, Mekelweg 2, 2628CD Delft,

Netherlands; tel. 31-152782198; fax 31-15-

2786730; e-mail

S.E.Offerman@

tudelft.nl.

Offerman is an assistant professor in the

Department of Materials Science and Engineering at Delft University of

Technology in the

Netherlands. His main research interests include solid-state nucleation and growth of grains/precipitates during phase transformations and precipitation reactions in metals, the development of fire-resistant steel, and experimental techniques related to neutron scattering and synchrotron radi-

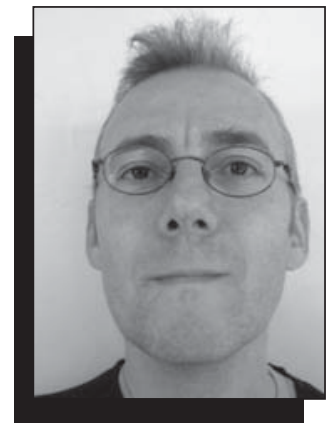

Eric Maire

ation. Offerman has received several Dutch talent grants and has more than 25 articles in international scientific journals.

David J. Rowenhorst can be reached by telephone at 202-767-2565; e-mail david.rowenorst@ nrl.navy.mil.

Rowenhorst is a staff scientist at the U.S. Naval Research Laboratory. He received his BS degree in 1999 from the University of Minnesota and his PhD degree in 2004 from Northwestern University. Afterward, Rowenhorst joined the Materials Science

Division at the U.S.

Naval Research

Laboratory as a postdoctoral fellow and was promoted to staff scientist in 2006. His research interests include 3D reconstruction, and analysis and visualization of microstructures, as well as the study of microstructural evolution and phase transformations.

Luc Salvo can be reached by e-mail at luc.salvo@ gpm2.inpg.fr.

Salvo is a lecturer at Grenoble INP in France.

He works mainly on microstructural evolution and relationships between microstructure and mechanical properties or dense $\mathrm{Al}$ alloys and metal foams. For this

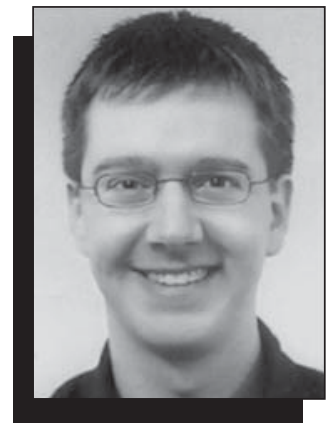

S. Erik Offerman

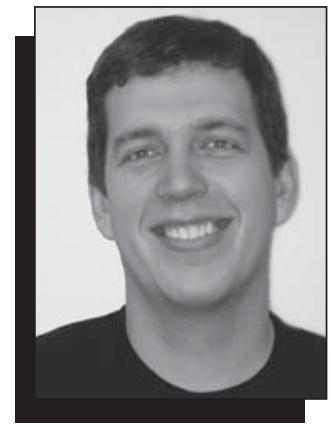

David J. Rowenhorst

202-767-5799; e-mail george.spanos@nrl.navy. mil.

Spanos is a section head at the Materials Science Division of the Naval Research Laboratory. He received his BS degree in 1982, his ME degree in 1985, and

his $\mathrm{PhD}$ degree in 1989-all in metallurgical engineering and materials science from Carnegie Mellon University. In 1989 , Spanos joined the Materials Science Division of the Naval Research Laboratory as a staff scientist and was promoted to his current position in 1994.

His research interests include the study of the kinetics, thermodynamics, morphological evolution, and overall mechanisms of solidstate phase transformations, the 3D analysis of microstructures, and the investigation of processing-microstructure -property relationships developed during the joining of materials. Spanos is the author of 75 technical articles in these research areas. In addition, he is a fellow of ASM International, a key reader of Metallurgical and Materials Transactions, and a member of various committees of The Minerals, Metals, \& Materials Society and ASM. 


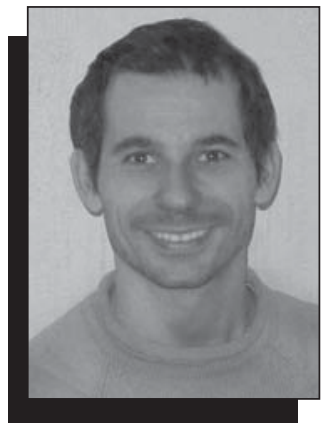

Luc Salvo

Peter W. Voorhees can be reached at the Department of Materials Science and Engineering, Northwestern University,

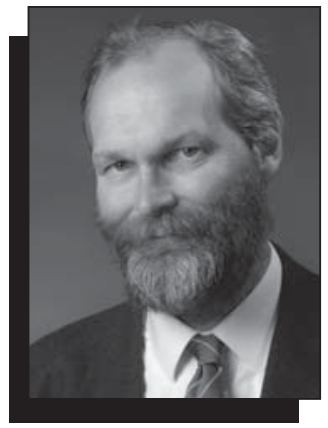

Jilt Sietsma

2220 Campus Dr.,

Evanston, IL 60208, USA; tel. 847-491-7815; e-mail p-voorhees@

northwestern.edu.

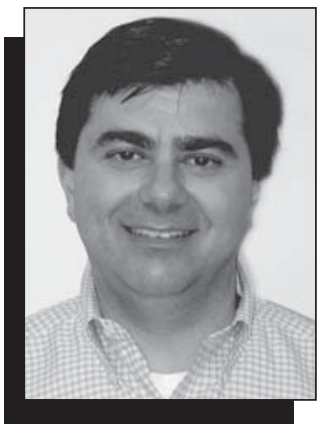

George Spanos

Voorhees is the Frank C. Engelhart Professor and chair of the

Department of Materials Science and Engineering

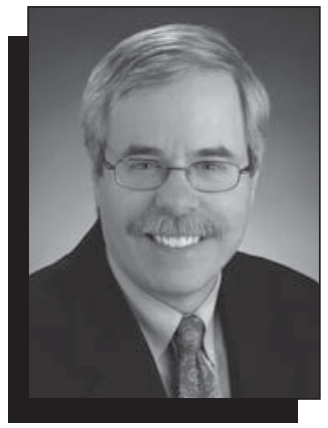

Peter W. Voorhees

at Northwestern

University. He received his $\mathrm{PhD}$ degree in materials engineering from Rensselaer Polytechnic
Institute. Voorhees' research interests include the dynamics of coarsening processes, nanowire growth, solid-oxide fuel cells, and the morphology of interfaces in materials. $\mathrm{He}$ also is a fellow of ASM International and the American Physical Society, and is listed as a highly cited researcher by the Institute for Scientific Information.

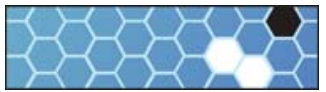

\section{Advertisers in This Issue}

Page No.

Page No.

\begin{tabular}{|c|c|c|c|}
\hline Bruker AXS, Inc. & Inside back cover & Janis Research Company, Inc. & $\overline{595}$ \\
\hline High Voltage Engineering Europa B.V. & Inside front cover & Microscopy Society of America & 595 \\
\hline Huntington Mechanical Laboratories, Inc. & Outside back cover & National Electrostatics Corp. & 602 \\
\hline
\end{tabular}

For free information about the products and services offered in this issue, check http://www.mrs.org/bulletin_ads

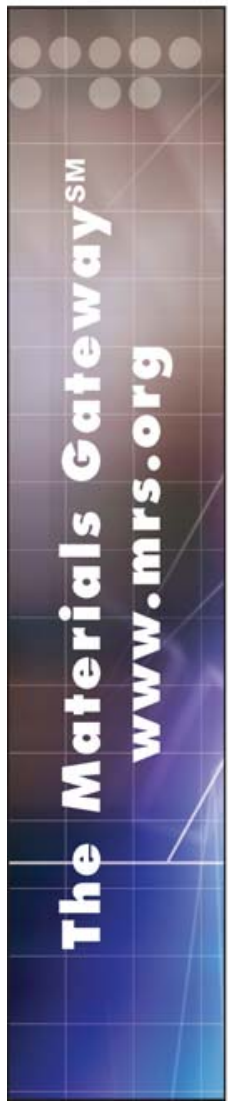

\section{Intensive Courses in Microscopy}

The Microscopy Society of America is offering intensive courses in:

- Nanomaterials Microscopy and Microanalysis: Tools and Preparation;

- Theory and Techniques of Aberration-Corrected Microscopy;

- Basic Principles of Confocal Microscopy.

Get 12 hours of instruction over 4 halfdays, during the "Microscopy and Microanalysis 2008" meeting in Albuquerque, August 3-7. See the world's largest exhibit of microscopy and microanalysis equipment, and attend scientific symposia, poster sessions, tutorials and other educational and social events. Join the largest annual microscopy gathering in the world!

For registration and details, please see: http://mm2008.microscopy.org/

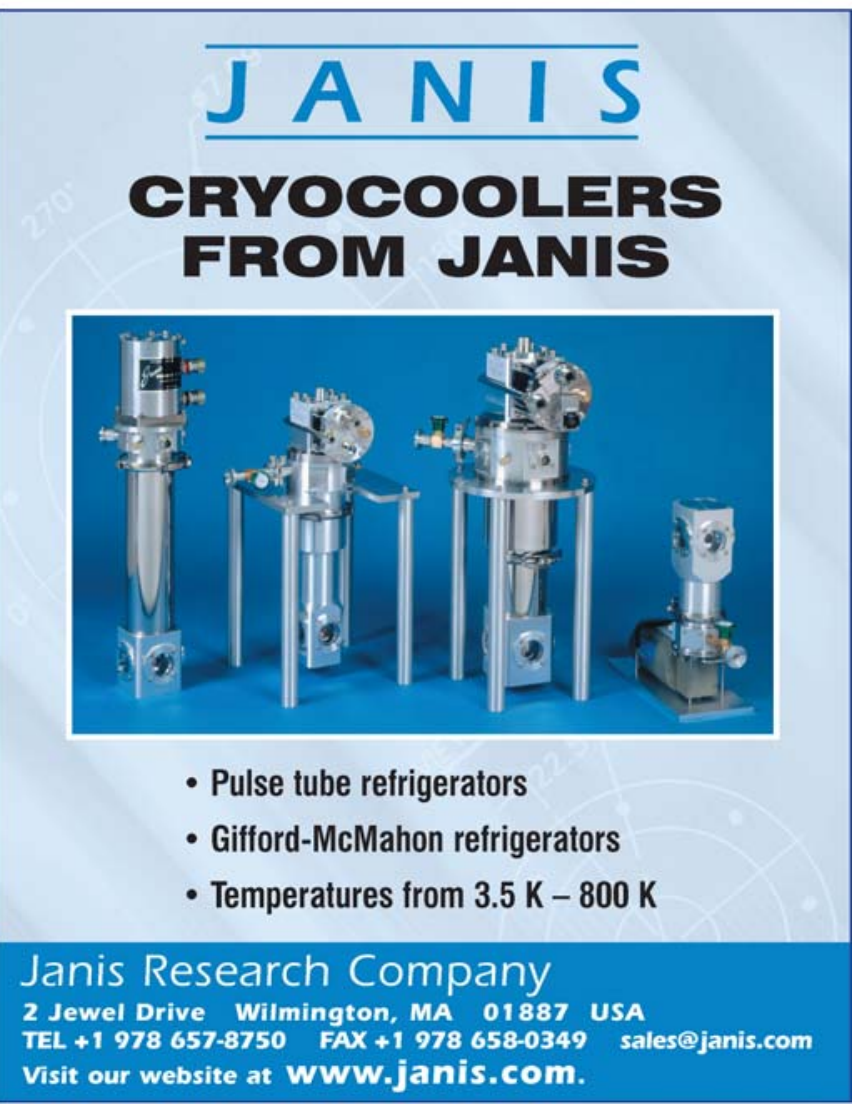

